\title{
Minimum-Light Phenomena in Nova-like Variables
}

\author{
GEORGE S. MUMFORD (Medford, Tucson)
}

\begin{abstract}
Some characteristics of the small-amplitude, quasi-periodic light fluctuations common to novalike variables are described. There appears to be a weak correlation for the majority of the objects between the dispersion about the mean magnitude and the interval between outbursts such that systems with infrequent outbursts exhibit the largest dispersions.

\section{Introduction}

Numerous observers have noted that novalike variables, including old, recurrent, and dwarf novae, display considerable variation in brightness at minimum light. For many of these objects, attempts have been made to establish whether or not these fluctuations are periodic, and to assess their physical characteristics. During the past few years, as a by-product of a search for eclipsing binaries among novalike variables, I have accumulated a fairly systematic body of data that can be used to study the variations in the so-called flickering for a fairly large sample of these stars. Some preliminary results are reported here.
\end{abstract}

\section{Observations}

All the photometric observations were obtained at Kitt Peak National Observatory using a dry-ice refrigerated $1 \mathrm{P} 21$ photomultiplier tube in conjunction with a 36-inch telescope. Continuous series of observations were made using 10-second integration and a blue filter. Individual deflections were converted to magnitude differences on the instrument system in the sense variable minus comparison, reduced to no atmosphere. Three color observations are based on several integrations in the various colors, and were reduced to the standard system through the usual procedures. Pertinent data are given in Table 1.

Table I: Observations of Novalike Variables

\begin{tabular}{|c|c|c|c|c|c|}
\hline Star & $B-V$ & $\mathbf{U}-\mathbf{B}$ & $\begin{array}{l}\text { Orbital Period } \\
\quad \text { (Hour) }\end{array}$ & $\begin{array}{l}\text { Cycle } \\
\text { (Day) }\end{array}$ & $\begin{array}{c}\text { Mean } \\
\text { Dispersion }\end{array}$ \\
\hline RX And & +0.10 & -0.86 & 5 h. & $14 \mathrm{~d}$ & 0.037 \\
\hline SS Aur & +0.12 & -1.01 & 4.3 & 55 & .217 \\
\hline $\mathrm{ZCam}^{+}$ & +0.55 & -0.77 & 7.0 & 23 & .109 \\
\hline SS Cyg & +0.26 & -1.01 & 6.6 & 50 & .161 \\
\hline $\mathrm{EM} \mathrm{Cyg}^{+}$ & +0.17 & -0.72 & 7.0 & 20 & .089 \\
\hline AB Dra & +0.96 & +0.01 & & 10 & .100 \\
\hline U Grem+ & +0.20 & -1.00 & 4.2 & 103 & .064 \\
\hline DQ Her ${ }^{+}$ & 0.00 & -0.88 & 4.7 & $?$ & .096 \\
\hline $\mathrm{AH}$ Her & +0.39 & -0.82 & & 20 & .078 \\
\hline N Her 1963 & -0.01 & -0.96 & & & .045 \\
\hline EX Hya ${ }^{+}$ & -0.07 & -1.09 & 1.6 & & .151 \\
\hline DI Lac & +0.24 & -0.64 & & & .053 \\
\hline T Leo & -0.07 & -1.06 & & & .185 \\
\hline X Leo & & & & 22 & .100 \\
\hline TU Leo & +0.56 & -0.08 & & & .051 \\
\hline CN Ori & +0.17 & -0.80 & & & .177 \\
\hline RU Peg & +0.65 & -0.43 & 8.9 & 70 & .081 \\
\hline TZ Per & +0.51 & -0.67 & & 17 & .048 \\
\hline GK Per & +0.71 & -0.58 & 16.5 & $?$ & .063 \\
\hline su UMa & +0.18 & -1.04 & & 16 & 0.198 \\
\hline
\end{tabular}

+ Exhibits eclipse-associated phenomena. 
In this table, the second and third columns refer to the mean colors determined from several nights of observation. The orbital periods are the photometric ones if the object exhibits eclipses, otherwise they are the spectroscopic ones (MUMFORD 1967). The cycle is the average interval between outbursts as gleaned from various reports of the American Association of Variable Star Observers. Of all the data these likely suffer the greatest ladk of completeness and systemization. In the final column, the dispersions about the mean magnitudes, averaged over several observing runs are listed. An attempt has been made to eliminate from these the effect of eclipses or phenomena associated with them, such as preand post-eclipse increases in brightness. Probably the indicated orders of magnitude are correct, though a considerable range of dispersions was found in many cases. Dispersions of SS Aurigae, for example, ranged from .170 to .308 over comparable periods of observation (MUMFORD 1966), when this star was near minimum brightness.

\section{Discussion}

More or less continuous series of observations on SU Ursae Majoris, TZ Persei, and DQ Herculis are shown in Figure 1. These may well serve as examples of the three rather different types of flickering observed. Bursts of several hundredths of a magnitude, separated in time by a few minutes, appearing superposed on an almost cyclical increase in brightness of the system are characteristic of the variations of SU Ursae Majoris. The amplitude is far smaller for TZ Persei and the interval between major bursts is longer; on the other hand, while the amplitude is small for DQ Herculis, the short interval between bursts is strictly periodic (WALKER 1961).

Because of the lack of adequate time resolution, it was decided, as a first approximation, to choose the average of the dispersion about the mean magnitude on particular nights as the parameter to describe the flickering.

Color indices are available for all but one of the objects listed in Table 1. These are plotted against the mean dispersion in the top two diagrams of Figure 2. In general, the bluest stars that are also the brightest in ultraviolet light exhibit the largest dispersions. Red systems apparently have only small dispersions.

Orbital periods based on photometric or spectroscopic observations are available for 10 objects. These are shown plotted against the mean dispersion in the third diagram from the top in Figure 2. One might expect that the closer together a pair of stars is, the greater their interaction, and, thus, possibly the dispersion might be. A least-squares line can be fitted to the data presented here indicating that such a trend may exist. However, if GK Persei, which weighs heavily in this solution, is omitted, this trend virtually disappears. But effects of orbital inclination may also be present here for we are considering a mix of eclipsing and non-eclipsing binaries.

The average interval in days between outbursts is available for 12 of the dwarf novae listed in Table 1, if U Geminorum and RU Pegasi, both with relatively small dispersions and long intervals between outbursts are excluded from consideration. There appears to be a relation between these two quantities as shown by the bottom diagram in Figure 2, such that objects of high dispersion have a long interval between outbursts. Besides the exceptions already noted, SU Ursae Majoris also departs markedly from the general trend.

It is anticipated that this study will be extended in the near future to include stars from the Southern Hemisphere for which some data already exist (MUMFORD 1971). Further, it would seem worthwhile to reanalyze the data on which the mean cycle between outburst is based, particularly for those systems of known orbital periods. Such an analysis might remove certain discrepancies described here as well as allow one to see if a connection between the two quantities exists.

This work has been supported by grants from the National Science Foundation.

$$
\text { References: }
$$

MUMFORD, G. S., 1966, Ap. J. 146, 411.

MUMFORD, G. S., 1967, P.A.S.P. 79, 283.

MUMFORD, G. S., 1971, Ap. J. 165, 369.

WALKER, M. F., 1961, Ap. J., 134, 171. 


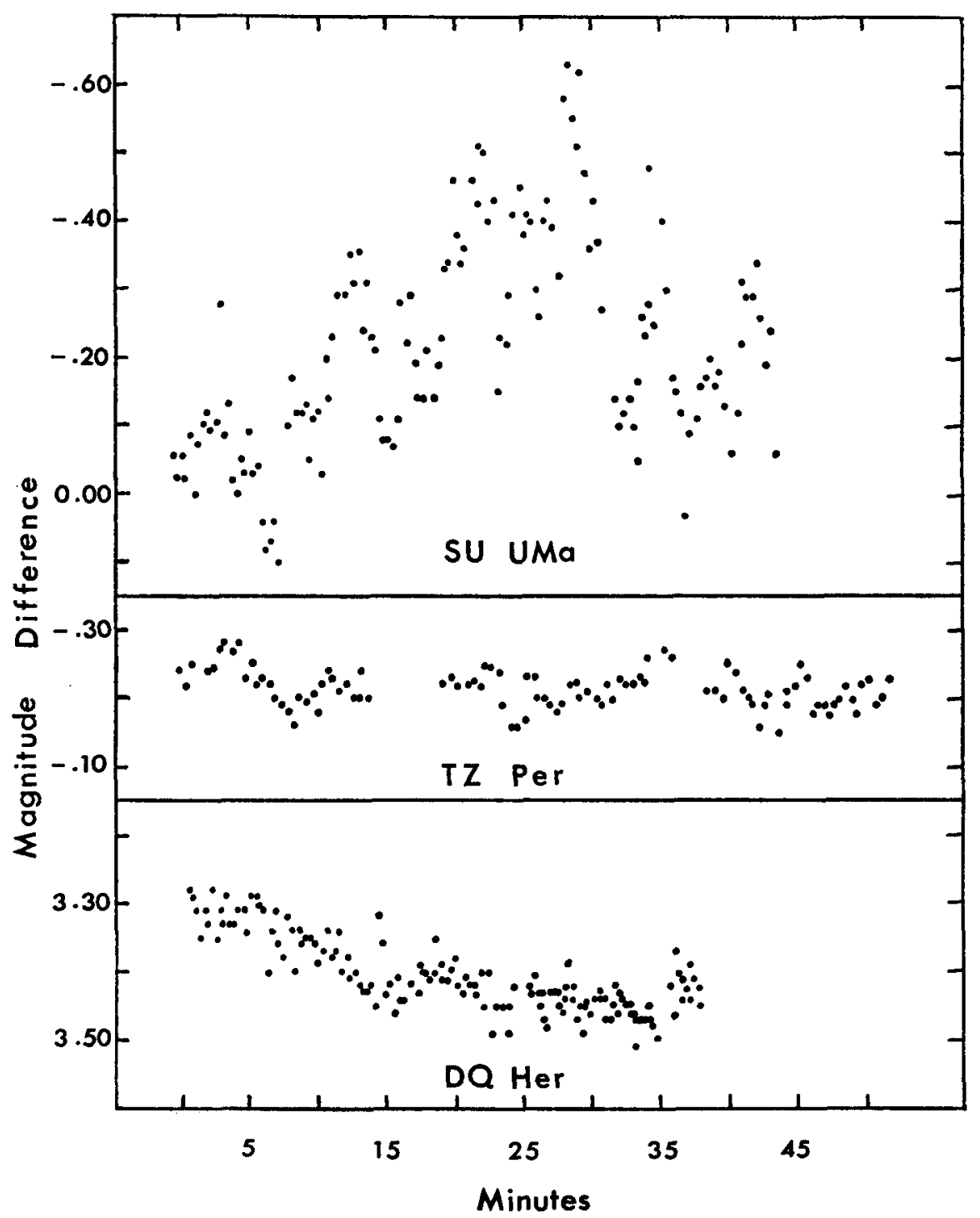

Fig. 1: Sample light curves of the novalike variables named. Ordinate is the blue magnitude difference in the sense variable minus comparison on the instrumental system; abscissa is time in minutes with zero arbitrary. 


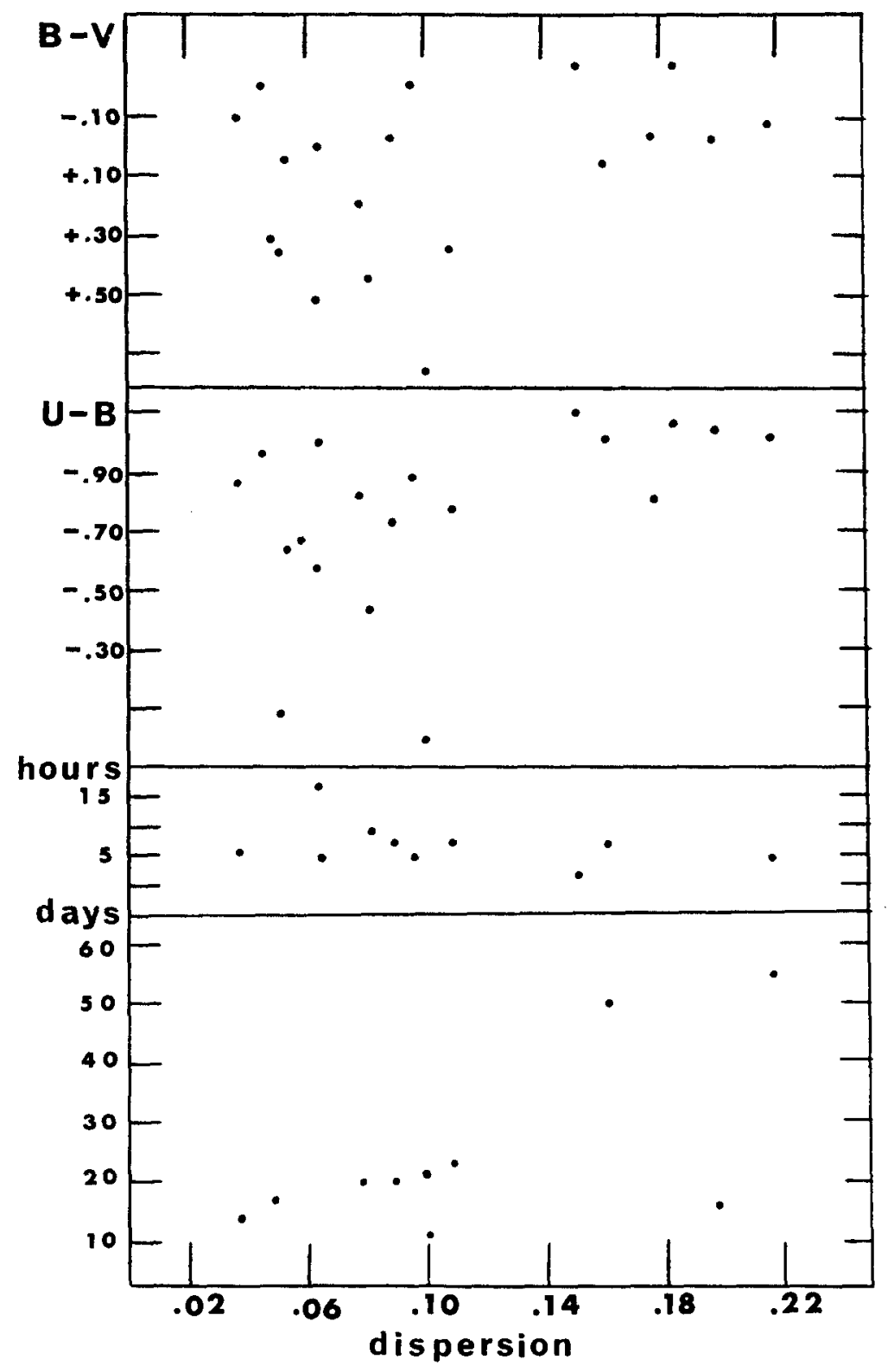

Fig. 2: Some characteristics of the flickering of novalike variables. Abscissa is the dispersion about the mean magnitude averaged over several runs; ordinates are, from top to bottom, the $\mathrm{B}-\mathrm{V}$ color index; the $\mathrm{U}-\mathrm{B}$ color index; the orbital period in hours; the interval between outbursts in days. Note that because of the scale used in the bottom diagram points representing U Geminorum and RU Pegasi do not appear. 